\title{
Looking for appropriateness in the cure of mixed vaginitis: the role of fenticonazole as an empiric treatment
}

\author{
Fabio Tumietto*,1, Brunella Posteraro ${ }^{2,3}$ \& Maurizio Sanguinetti ${ }^{4,5}$ \\ ${ }^{1}$ Infectious Diseases Unit Teaching Hospital, S Orsola-Malpighi, Alma Mater Studiorum, University of Bologna, Bologna, Italy \\ ${ }^{2}$ Fondazione Policlinico Universitario A Gemelli IRCCS, Dipartimento di Scienze Gastroenterologiche, Endocrino-Metaboliche e \\ Nefro-Urologiche, Rome, Italy \\ ${ }^{3}$ Università Cattolica del Sacro Cuore, Istituto di Patologia Medica e Semeiotica Medica, Rome, Italy \\ ${ }^{4}$ Fondazione Policlinico Universitario A Gemelli IRCCS, Dipartimento di Scienze di Laboratorio e Infettivologiche, Rome, Italy \\ ${ }^{5}$ Istituto di Microbiologia, Università Cattolica del Sacro Cuore, Rome, Italy \\ *Author for correspondence: Fabio.tumietto@unibo.it
}

\begin{abstract}
Mixed vaginitis is defined as the simultaneous presence of at least two different vaginal pathogens, both contributing to an abnormal vaginal milieu leading to signs and symptoms. Pathogen coinfection occurs frequently in women with vaginitis, and both coinfection and mixed vaginitis have relevant clinical and therapeutic implications. Fenticonazole, an imidazole derivative with a broad spectrum of antimycotic and antimicrobial activity, appears at least as effective as other topical antifungals in the treatment of vulvovaginal candidiasis and can also have a major role in the treatment of mixed infections or coinfections of the lower genital tract. This paper will address the current role of topical fenticonazole as an empiric treatment of vulvovaginal infections, with a focus on the effectiveness in the treatment of mixed vaginitis and the possible implications of this.
\end{abstract}

First draft submitted: 20 June 2019; Accepted for publication: 8 November 2019; Published online: 17 December 2019

Keywords: fenticonazole $\bullet$ fungal infection $\bullet$ mixed infection $\bullet$ vulvovaginal candidiasis

Vulvovaginal candidiasis are superficial infections, limited to mucosa, without deep involvement of organs [1]. They represent treatable, but very frequent conditions; among different types of infections, Candida vulvovaginitis affects up to $75 \%$ of women at least once in their lifetime and can be associated with severe clinical outcomes [2,3]. Recurrent infection by Candida, or recurrent vulvovaginal candidiasis, is also common, with a global annual prevalence of 3871/100,000 women [4]. Candida albicans is the most frequent pathogenic agent [5]. However, infections by non- $C$. albicans, especially Candida glabrata, Candida parapsilosis and Candida tropicalis, are increasingly reported [6].

Mixed vaginitis is defined as the simultaneous presence of at least two different vaginal pathogens, both contributing to an abnormal vaginal milieu leading to signs and symptoms [7]. Notably, both pathogens require specific therapy for complete eradication. In coinfection, although two pathogens are identified, a potential pathogen may be present but may not be a cause of existing vaginal symptoms. Approximately $20-30 \%$ of women with bacterial vaginosis are coinfected by the Candida species, and coexistence of bacterial pathogens and Trichomonas vaginalis is also often reported (coinfection rates of 60-80\%), both with significant clinical and therapeutic implications [7]. Indeed, it has been reported how the interaction between Candida and bacteria species may influence the antimicrobial susceptibility of both organisms [8].

Treatment of Candida infections largely depends on the use of azole (imidazole/triazole) or polyene antifungals, administered topically in short courses or orally for longer periods, according to the severity and site of the infection [1]. Of note, antimicrobial resistance is a worldwide issue, with large clinical and economic impact. Upon the selective pressure by antifungals, the increased prevalence of non- $C$. albicans strains, endowed with variable susceptibility and even full resistance to azoles, may occur [1].

Fenticonazole is an imidazole derivative with a broad spectrum of antimycotic and antimicrobial activity and appears to be at least as effective as other topical antifungals in the treatment of vulvovaginal candidiasis and can 
also have a major role in the treatment of mixed vaginal infections or coinfections of the lower genital tract $[1,9]$. This paper will address the current role of topical fenticonazole as an empiric treatment of vulvovaginal infections, with a focus on effectiveness in the treatment of mixed vaginitis and the possible implications of this.

\section{Overview of pharmacological properties}

Fenticonazole (alpha-[2,4-dichlorophenyl]-beta,N-imidazolylethyl 4-phenylthiobenzyl ether nitrate) is an imidazole derivative specifically developed for the topical treatment of fungal infections. This molecule presents a wide spectrum of activity against different dermatophytes and yeasts, by different mechanisms, mainly by damaging the fungal cell membrane via inhibition of the fungal CYP450 isozyme lanosterol 14-alpha-demethylase, which is required to convert lanosterol to ergosterol, an essential component of fungal cell membrane synthesis [9]. Another mechanism involves the inhibition of the production of secreted acid (aspartic) protease by C. albicans, a specific enzyme that has been correlated with the adherence of the yeast to epithelial cells $[10,11]$. Remarkably, aspartic protease has been recently identified as a major contributor to Candida virulence as it is involved in adhesion, invasion and tissue damage. Indeed, new molecules specifically targeting this enzyme are being developed [1,12-14]. Fenticonazole also exerts notable antibacterial activity against Gardnerella vaginalis and Gram-positive bacteria (e.g., Staphylococcus aureus, Staphylococcus epidermidis, Streptococcus spp.), which are often reported in vaginal infections $[15-17]$ and is active against $T$. vaginalis $[18,19]$. Very recent evidence shows the activity of fenticonazole against Leishmania species [20].

Remarkably, fenticonazole can be retained in the stratum corneum of the skin for a long time, and it is endowed by peculiar pharmacokinetic properties making it accumulate in the mucosal tissue as an active drug for up to $72 \mathrm{~h}$. Together, these properties allow the formation of a local reservoir of fenticonazole when administered topically and delay consecutive administrations [1]. Furthermore, fenticonazole is poorly absorbed systemically: this contributes to its favorable safety profile without interfering with the gastrointestinal microbiome $[1,21,22]$. All these properties make fenticonazole a drug of choice for the empirical treatment of vulvovaginitis when used before the confirmation of a definitive diagnosis or when the identification of the specific infective agent cannot be promptly performed.

\section{Clinical efficacy in vaginal infections due to Candida alone}

In the gynecological setting, fenticonazole was studied in the treatment of Candida (vulvo)vaginitis [23-33]. In all studies, the diagnosis was based on clinical history and presentation, as well as direct mycological examinations and cultures. A detailed description of these studies goes beyond the aims of the present review and can be found in [1].

\section{Clinical efficacy in bacterial or mixed vaginal infections \\ Bacterial vaginitis}

The efficacy of fenticonazole in the treatment of aerobic bacterial vaginitis has been evaluated in a pilot, prospective, randomized, double-blind, placebo-controlled study on 47 women [34]. Fenticonazole was administered as 2\% vaginal cream, $5 \mathrm{~g}$ placed high in the vagina for 7 days. The overall cure rate with fenticonazole group was 85 versus $35 \%$ with placebo $(\mathrm{p}=0.003)$. The recurrence rate was lower with fenticonazole $(11.8 \mathrm{vs} 50 \%)$. The safety profile of fenticonazole was comparable with that of placebo.

\section{Mixed vaginitis}

Noteworthy, C. albicans and S. aureus can coexist in polymicrobial biofilms [35]. Staphylococcus aureus attaches strongly to hyphae of $C$. albicans, and hyphae-associated $S$. aureus become less susceptible to antibiotic treatment. Furthermore, co-inoculation of $C$. albicans and $S$. aureus causes more severe and widespread infection than either microorganism alone. Interaction of $C$. albicans with $S$. aureus or other bacterial species influences the antimicrobial susceptibility of both organisms when evaluated in vitro, mimicking the ecology of mixed vaginal infection exposed to antibiotics [8].

The efficacy of fenticonazole in mixed bacterial/Candida infection has been investigated in four clinical studies $[28,31,36,37]$. Scalambrino $e$ al. conducted a study in 67 patients to evaluate therapeutic effect of fenticonazole $1000 \mathrm{mg}$ administered as a single dose in vaginal capsules + vaginal wash for five consecutive days in the treatment of vaginal trichomoniasis, with or without concomitant candidiasis. The study demonstrated that fenticonazole eradicated all organisms in 100\% of patients at 10-12 days and only two patients had a subclinical infection at the subsequent follow-up visit; tolerability was judged as 'excellent' [31]. In one open-label study involving women with T. vaginalis and vaginal mixed infections treated with a single ovule of fenticonazole $600 \mathrm{mg}, 96 \%(55 / 57)$ of 
Table 1. MIC $_{90}$ of 318 vaginal isolates to fenticonazole (as determined by the EUCAST broth microdilution method).

\begin{tabular}{|l|l|}
\hline Species (number of isolates) & $\mathrm{MIC}_{90}, \mu \mathrm{g} / \mathrm{ml}$ (range) \\
\hline Escherichia coli (25) & $0.125(0.016-0-25)$ \\
\hline Streptococcus agalactiae (30) & $0.03(0.008-0.125)$ \\
\hline Staphylococcus aureus (35) & $1(0.25-4)$ \\
\hline Candida albicans (51) & $0.06(0.016-0.25)$ \\
\hline Candida glabrata (44) & $0.25(0.06-0.5)$ \\
\hline Candida tropicalis (39) & $0.125(0.03-0.25)$ \\
\hline Candida parapsilosis (52) & $0.03(0.008-0.125)$ \\
\hline Gardnerella vaginalis (42) & $0.03(0.008-0.03)$ \\
\hline MIC: Minimum inhibitory concentration. & \\
\hline Adapted with permission from [8]. & \\
\hline
\end{tabular}

patients eradicated C. albicans, 67\% eradicated T. vaginalis and 45\% were negative for all organisms at day 7 [36]. More recently, in a multicenter, prospective, open-label study, fenticonazole $(1 \mathrm{~g}$ vaginal suppository once daily on days 1 and 3) was evaluated in 101 women with vulvovaginitis involving single or mixed infections with $C$. albicans, T. vaginalis and/or G. vaginalis [37]. At day 8, the rate of eradication of C. albicans was $90 \%$ (26/29), 70\% for $T$. vaginalis and $67 \%$ for $G$. vaginalis; 28 days later, no recurrence was reported in either the C. albicans or T. vaginalis groups. In patients presenting with mixed infections, eradication of $C$. albicans was achieved in $90 \%$, eradication of T. vaginalis was achieved in $62 \%$, eradication of $G$. vaginalis was achieved in $59 \%$, and eradication of all pathogens involved was achieved in $45 \%$ of patients. Overall, eradication of all offending pathogens was achieved in $67 \%$ of the total population, with a relapse rate of only $16 \%$. Signs and symptoms of infections significantly improved at day 8 versus baseline $(\mathrm{p}<0.05)$. Schneider et al. investigated the therapeutic activity of a single fenticonazole $600 \mathrm{mg}$ ovule in 72 women either with vaginal candidiasis alone or in association with other infective agent apart from Trichomonas. The symptom scores for erythema, itching, discharge and edema decreased by more than $90 \%$ within 1 week of treatment initiation and mycological/microbiological findings were negative in $76 \%$ of cases at the first follow-up visit (day 7 after treatment). There was no report of any systemic or local adverse reaction in the study [28].

Despite the extensive clinical experience collected to date, so far, the evaluation of fenticonazole antimicrobial activity did not include time-kill assays using either single or mixed cultures of Candida species $[8,38]$. In order to address this issue, we have recently investigated, for the first time to our knowledge, the dynamics of fenticonazoleinduced killing either in mono-species or dual-species testing assays for vaginal Candida and bacterial isolates, with a focus on mixed cultures [8]. Minimum inhibitory concentrations (MIC) ${ }_{90}$ values are reported in Table 1 [8]. Among 318 vaginitis isolates of Candida and bacterial species, 28 isolates were selected for time-kill curve studies. At concentrations equal to $8 \times \mathrm{MIC}, 99.9 \%$ killing was reached after $19 \mathrm{~h}$ for C. glabrata and $20 \mathrm{~h}$ for C. tropicalis, respectively (Table 2) [8]. Remarkably, at concentrations equal to $2 \times \mathrm{MIC}$, approximately $20 \mathrm{~h}$ were required to reach this end point against $C$. albicans in mixed culture with S. aureus, Streptococcus agalactiae or Escherichia coli. Overall, time-kill data highlighted that fenticonazole is microbicidal at supra-MIC concentrations, such as those easily achieved in topically treated womens' skin/mucosa surfaces during vaginitis episodes: fenticonazole when administered intravaginally penetrates into the vaginal mucosa generating, after a single administration, significant dose-independent tissue concentrations after $3 \mathrm{~h}$ (between 20 and $30 \mu \mathrm{g} / \mathrm{g}$ of tissue) and still relevant dose-dependent tissue levels after $12 \mathrm{~h}$, namely $2.8 \pm 1.3 \mu \mathrm{g} / \mathrm{g}$ after a single $200 \mathrm{mg}$ ovule administration, $3.9 \pm 1.8$ and $5.1 \pm 2.1 \mu \mathrm{g} / \mathrm{g}$ after single 600 and $1000 \mathrm{mg}$ ovules administration, respectively, concentrations sufficient to obtain significant 'in vitro' growth reduction for all the tested pathogens [39]. The fungicidal effect of fenticonazole in three mixed cultures of $C$. albicans $+S$. aureus, $C$. albicans $+S$. agalactiae and $C$. albicans + E. coli was similar to that of the pure cultures of $C$. albicans (Figure 1) [8]. Although the elucidation about the mechanism behind the fenticonazole antibacterial activity requires further investigation, induction of a common oxidative-damage cellular death pathway that culminates in the formation of lethal reactive oxygen species, as observed for other molecules belonging to the imidazole class, can be postulated [8]. Moreover, other mechanisms including a direct bacterial membrane damage caused by the imidazole can also be speculated [40]. 
Table 2. Mean times to achieve $99 \%$ or $99.9 \%$ growth reductions at the indicated multiple of the Minimum inhibitory concentration of fenticonazole.

\begin{tabular}{|c|c|c|c|c|c|c|}
\hline \multirow[t]{2}{*}{ Cultures } & \multicolumn{3}{|c|}{ t99\% } & \multicolumn{3}{|c|}{ t99.9\% } \\
\hline & $2 \times \mathrm{MIC}$ & $4 \times \mathrm{MIC}$ & $8 \times \mathrm{MIC}$ & $2 \times \mathrm{MIC}$ & $4 \times \mathrm{MIC}$ & $8 \times \mathrm{MIC}$ \\
\hline \multicolumn{7}{|l|}{ Pure cultures } \\
\hline Candida albicans & 11.56 & 11.31 & 12.85 & 17.34 & 16.95 & 19.28 \\
\hline Candida glabrata & NA & $>48$ & 12.39 & NA & $>48$ & 18.59 \\
\hline Candida parapsilosis & $>48$ & 11.53 & 12.41 & $>48$ & 17.30 & 18.62 \\
\hline Candida tropicalis & NA & NA & 13.61 & NA & NA & 20.41 \\
\hline $\begin{array}{l}\text { Staphylococcus } \\
\text { aureus }\end{array}$ & 8.06 & 7.06 & 6.27 & 12.10 & 10.60 & 9.41 \\
\hline $\begin{array}{l}\text { Streptococcus } \\
\text { agalactiae }\end{array}$ & 7.46 & 6.87 & 6.09 & 11.19 & 10.30 & 9.13 \\
\hline Escherichia coli & 7.43 & 6.87 & 6.26 & 11.15 & 10.31 & 9.40 \\
\hline \multicolumn{7}{|l|}{ Mixed cultures } \\
\hline Candida albicans plus & 13.87 & 10.45 & 12.36 & 20.81 & 15.68 & 18.54 \\
\hline $\begin{array}{l}\text { Staphylococcus } \\
\text { aureus }\end{array}$ & 7.71 & 6.80 & 5.74 & 11.57 & 10.19 & 8.62 \\
\hline Candida albicans plus & 13.48 & 12.39 & 11.79 & 20.22 & 18.58 & 17.68 \\
\hline $\begin{array}{l}\text { Streptococcus } \\
\text { agalactiae }\end{array}$ & - & 7.08 & 6.57 & - & 10.62 & 9.86 \\
\hline Candida albicans plus & 13.66 & 13.28 & 11.83 & 20.49 & 19.93 & 17.74 \\
\hline Escherichia coli & 7.71 & 6.80 & 5.74 & 11.57 & 10.19 & 8.62 \\
\hline
\end{tabular}

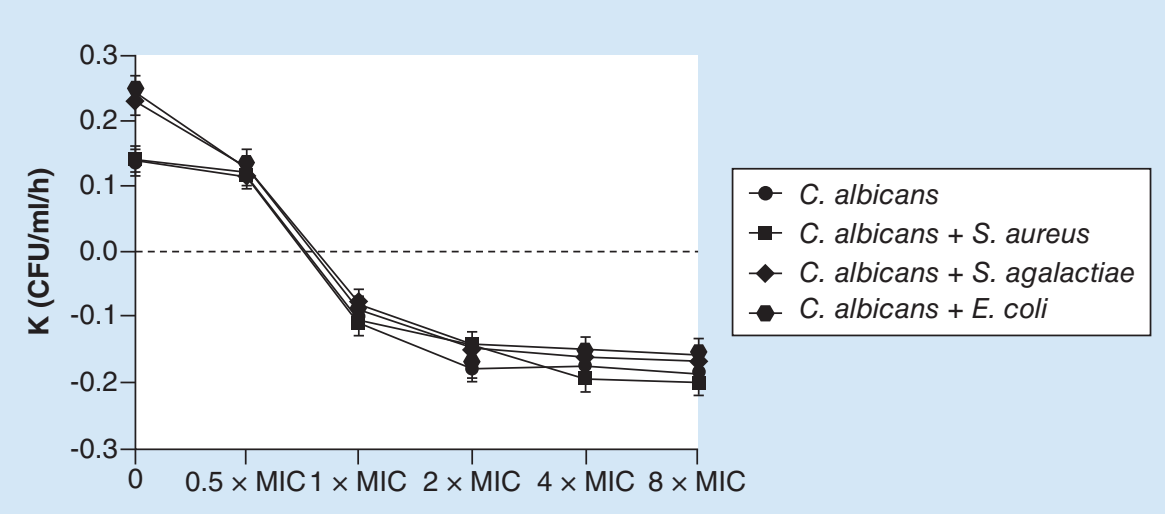

Figure 1. Fungicidal effect of fenticonazole in three mixed cultures. Killing rates (K) for mixed cultures of Candida albicans with either Staphylococcus aureus, Streptococcus agalactiae and Escherichia coli. Values above the broken lines indicate growth, and values below the broken lines indicate killing.

MIC: Minimum inhibitory concentration.

Reproduced with permission from [8].

\section{Implications for clinical practice}

Inappropriate treatment of uncomplicated infections can affect the ecological characteristics of pathogenic fungi and worsen the outcome of infections [1]. Worldwide, there is an increasing tendency to prescribe oral medications as a first-line therapy, although current guidelines indicate the equivalent topical therapy as preferred over oral treatment in superficial infections. Indeed, oral formulations do expose the intestinal microbiota to the action of the active compound, contributing to the selection of resistant strains. On the other hand, an effective topical treatment could contribute in reducing the prescription of systemic drug with a consequent reduction of perturbance of intestinal microbiota. Moreover, reducing prescription of sub-optimal dose of systemic antifungal drug does reduce the risk of selecting resistant strains. At last, many patients prefer topical treatment over systemic treatment [15]. 
The efficacy of fenticonazole as a topical alternative to multi-agent antimicrobial treatment of mixed bacterial and fungal infections has been shown in different clinical studies $[28,31,34,36,37]$. Furthermore, recently acquired time-kill data highlight the potential microbicidal activity of fenticonazole at supra-MIC concentrations, which can be easily reached in topically treated patients for skin/mucosa surfaces [8,39]. These data also show that increasing fenticonazole concentrations allows to overcome the potential interference between $C$. albicans and $S$. aureus or other bacterial species in mixed infections, thus reducing the risk of developing resistance. These findings have immediate relevance to clinical practice: indeed, given its wide spectrum of action and the ability to exert antimicrobial activity against fungi, bacteria and mixed infections, fenticonazole may be administered as a first-line therapy without the immediate need of performing a test to identify the pathogen. In order to further increase the efficacy of fenticonazole, the concomitant administration of probiotics as adjuvant therapy could increase the rate of short-term clinical and mycological cure and decrease the relapse rate at 1 month, without increasing the risk of adverse events [41].

Furthermore, our attention must be directed to the current availability of topical treatments based on the composition of different drugs. In this regard, the conclusions of a recent multicenter study comparing topical treatment with nystatin-neomycin-polymyxin B and miconazole alone, an azole derivative that in many respects can be compared with fenticonazole, have been recently reported. This study did not demonstrate a superiority of the combination treatment. No difference was observed between the two treatments, therefore the first-line treatment with the combination nystatin-neomycin-polymyxin B for 12 days is equivalent to the empirical treatment with 3 days of imidazole derivative without apparent advantages, and with all the disadvantages of such a long treatment [42]. On these bases, the use of topical fenticonazole can be recommended for the first-line, empiric treatment of vaginal and mixed infections with different etiology, minimizing the risk of selecting drug-resistant microbial strains and avoiding perturbance of intestinal microflora [1].

\section{Conclusion}

Vulvovaginal candidiasis is a very frequent condition, often associated with bacterial infections. The mixed vaginitis has a significant clinical and therapeutic implications.

Clinical evidence on the efficacy and the safety of fenticonazole has been reported, showing both a broad spectrum of antimycotic and antimicrobial activity, and a safe and tolerable profile that allow its use as a first-line empiric treatment of mixed vaginitis.

\section{Executive summary}

- Inappropriate treatment of uncomplicated infections can affect the ecological characteristics of pathogenic fungi and worsen the outcome of infections. Worldwide, there is an increasing tendency to prescribe oral medications as a first-line therapy.

- On the other hand, an effective topical treatment could contribute in reducing the prescription of systemic drug with a consequent reduction of perturbance of intestinal microbiota. Moreover, reducing prescription of sub-optimal dose of systemic antifungal drug does reduce the risk of selecting resistant strains.

Clinical efficacy

- The efficacy of fenticonazole as a topical alternative to multi-agent antimicrobial treatment of mixed bacterial and fungal infections has been shown in different clinical studies.

- Furthermore, recently acquired time-kill data highlight the potential microbicidal activity of fenticonazole at supra-minimum inhibitory concentrations, such those easily achieved in topically treated skin/mucosa surfaces during vaginitis episodes.

- These data also show that increasing fenticonazole concentrations allows to overcome the potential interference between Candida albicans and Staphylococcus aureus or other bacterial species in mixed infections, thus reducing the risk of developing resistance.

- Given its wide spectrum of action and the ability to exert antimicrobial activity against fungi, bacteria and mixed infections, fenticonazole may be administered as a first-line therapy without the immediate need of performing a test to identify the pathogen.

Conclusion

- The use of topical fenticonazole can be recommended for the first line, empiric treatment of vaginal and mixed infections with different etiology, minimizing the risk of selecting drug-resistant microbial strains and avoiding perturbance of intestinal microflora. 
This approach represents a relevant alternative to systemic multi-agent antimicrobial treatment of mixed bacterial and fungal, reducing the risk of microbial resistance and of perturbance of intestinal microbiota.

Financial \& competing interests disclosure

The authors have no relevant affiliations or financial involvement with any organization or entity with a financial interest in or financial conflict with the subject matter or materials discussed in the manuscript. This includes employment, consultancies, honoraria, stock ownership or options, expert testimony, grants or patents received or pending, or royalties.

Editorial assistance was provided by L Giacomelli and A Shah (Polistudium, Milan, Italy); graphical assistance was provided by M Pianta, on behalf of Polistudium. These were supported by Recordati.

\section{References}

Papers of special note have been highlighted as: $\bullet$ of interest; $\bullet \bullet$ of considerable interest

1. Tumietto F, Giacomelli L. Fenticonazole: an effective topical treatment for superficial mycoses as the first-step of antifungal stewardship program. Eur. Rev. Med. Pharmacol. Sci. 21(11), 2749-2756 (2017).

-• Recent review summarizing available studies on fenticonazole.

2. Sobel JD. Vulvovaginal candidosis. Lancet 369(9577), 1961-1971 (2007).

-• Review on the epidemiology and pathogenesis of this infection, also discussing its management strategies.

3. Tedeschi S, Tumietto F, Giannella $\mathrm{M}$ et al. Epidemiology and outcome of candidemia in internal medicine wards: a regional study in Italy. Eur. J. Intern. Med. 34, 39-44 (2016).

4. Denning DW, Kneale M, Sobel JD, Rautemaa-Richardson R. Global burden of recurrent vulvovaginal candidiasis: a systematic review. Lancet Infect. Dis. 18, e339-e347 (2018).

- Excellent paper assessing the burden of recurrent vulvovaginal candidiasis

5. Donders GG, Sobel JD. Candida vulvovaginitis: a store with a buttery and a show window. Mycoses 60, 70-72 (2017).

6. Gonçalves B, Ferreira C, Alves CT, Henriques M, Azeredo J, Silva S. Vulvovaginal candidiasis: epidemiology, microbiology and risk factors. Crit. Rev. Microbiol. 42(6), 905-927 (2016).

7. Sobel JD, Subramanian C, Foxman B, Fairfax M, Gygax SE. Mixed vaginitis-more than coinfection and with therapeutic implications. Curr. Infect. Dis. Rep. 15(2), 104-108 (2013).

8. Sanguinetti M, Cantón E, Torelli R, Tumietto F, Espinel-Ingroff A, Posteraro B. In vitro activity of fenticonazole against Candida and bacterial vaginitis isolates determined by mono- or dual-species testing assays. Antimicrob. Agents Chemother. 63(7), pii: e02693-18 (2019).

- Recent in vitro study on fenticonazole, which represents the basis for the present paper.

9. Veraldi S, Milani R. Topical fenticonazole in dermatology and gynaecology: current role in therapy. Drugs 68, 2183-2194 (2008).

10. Angiolella L, De Bernardis F, Bromuro C, Mondello F, Ceddia T, Cassone A. The effect of antimycotics on secretory acid proteinase of Candida albicans. J. Chemother. 2(1), 55-61 (1990).

11. De Bernardis F, Cassone A. [Comparison of the effects of fenticonazole and econazole on the aspartic proteinase secreted by Candida albicans]. Contracept. Fertil. Sex. 24(2), 163-165 (1996).

12. Monika S, Matgorzata B, Zbigniew O. Contribution of aspartic proteases in Candida virulence. Protease inhibitors against Candida infections. Curr. Protein Pept. Sci. 18(10), 1050-1062 (2017).

13. De Bernardis F, Graziani S, Tirelli F, Antonopoulou S. Candida vaginitis: virulence, host response and vaccine prospects. Med. Mycol. 56(Suppl. 1), 26-31 (2018).

14. Silva NC, Nery JM, Dias AL. Aspartic proteinases of Candida spp.: role in pathogenicity and antifungal resistance. Mycoses 57(1), 1-11 (2014).

15. Jones BM, Geary I, Lee ME, Duerden BI. Comparison of the in vitro activities of fenticonazole, other imidazoles, metronidazole, and tetracycline against and skin infections. Antimicrob. Agents Chemother. 33, 970-972 (1989).

16. Veronese M, Barzaghi D, Bertoncini A. Antifungal activity of fenticonazole in experimental dermatomycosis and candidiasis. Arzneimittelforschung 31(12), 2137-2139 (1981).

17. Veronese M, Barzaghi D, Bertoncini A, Zadro M. Mutagenicity studies on fenticonazole, a new antifungal imidazole derivative. Arzneimittelforschung 31(12), 2142-2144 (1981).

18. Manth SM, Rindt W, Schnitker J, Weigerding A. Fenticonazole in the treatment of vaginal trichomonas infections. Curr. Ther. Res. 45 , 1060-1066 (1989).

19. Fontana C, Cammarata E, Greco M, Oliveri S. In vitro activity of fenticonazole on Trichomonas vaginalis and Gardenerella vaginalis. Curr. Ther. Res. 48, 44-51 (1990). 
20. Yamamoto ES, Jesus JA, Bezerra-Souza A, Laurenti MD, Ribeiro SP, Passero LFD. Activity of fenticonazole, tioconazole and nystatin on new world Leishmania species. Curr. Top. Med. Chem. 18(27), 2338-2346 (2018).

21. Fioroni A, Terragni L, Vannini P et al. Fenticonazole plasma levels during treatment with fenticonazole $2 \%$ cream and spray in patients with dermatomycoses. Curr. Ther. Res 47, 997-1003 (1990).

22. Graziani G, Cazzulani P, Barbadoro E. Toxicological and pharmacological properties of fenticonazole, a new topical antimycotic. Arzneimittelforschung 31, 2145-2151 (1981).

23. Brewster E, Preti PM, Ruffm ann R, Studd J. Effect of fenticonazole in vaginal candidiasis: a double-blind clinical trial versus clotrimazole. Int. J. Med. Res. 14, 306-310 (1986).

24. Wiest W, Ruffmann R. Short-term treatment of vaginal candidiasis with fenticonazole ovules: a threedose schedule comparative trial. $J$. Int. J. Med. Res. 15, 319-325 (1987).

25. De Cecco L, Gorlero F, Marre Brunenghi MC, Venturini PL. Studio multicentrico sull'efficacia e tollerabilità del fenticonazolo nel trattamento delle vulvovaginiti da candida. Int. J. Drug Ther. 5, 296-301 (1988).

26. Studd JW, Dooley MM, Welch CC et al. Comparative clinical trial of fenticonazole ovule (600 mg) versus clotrimazole vaginal tablet $(500 \mathrm{mg})$ in the treatment of symptomatic vaginal candidiasis. Curr. Med. Res. Opin. 11, 477-484 (1989).

27. Wiest W, Azzollini E, Ruffmann R. Comparison of single administration with an ovule of $600 \mathrm{mg}$ fenticonazole versus a $500 \mathrm{mg}$ clotrimazole vaginal pessary in the treatment of vaginal candidiasis. J. Int. J. Med. Res. 17, 369-372 (1989).

28. Schneider D, Caspi E, Arieli S, Bukovski I. Fenticonazole in the treatment of vaginal candidiasis. Adv. Ther. 7, 355-361 (1990).

29. Lawrence AG, Houang ET, Hiscock E, Wells MB, Colli E, Scatigna M. Single dose therapy of vaginal candidiasis: a comparative trial of fenticonazole vaginal ovules versus clotrimazole vaginal tablets. Curr. Med. Res. Opin. 12, 114-120 (1990).

30. Zivaljevic B, Golubovic I, Seratlić J et al. Efficiency of fenticonazole for the treatment of vaginal candidiasis. Srp. Arhcelok. Lek. 140, 469-474 (2012).

31. Scalambrino S, Colombo M, Pellegrino A. Efficacy and tolerability of intravaginal fenticonazole single therapeutic shot in low-recurrent vaginal trichomoniasis and candidiasis. G. Ital. Mal. Inf. 2, 37-40 (1996) [Italian].

32. Malova IO. Efficacy of azole drugs in acute vulvovaginal candidiasis. Akusherstvo I Ginekologiya/Obstetrics Gynecology (10), 109-114 (2016) [Russian].

33. Murina F, Graziottin A, Felice R, Di Francesco S, Mantegazza V. [Short-course treatment of vulvovaginal candidiasis: comparative study of fluconazole and intra-vaginal fenticonazole]. Minerva Ginecol. 64, 89-94 (2012) [Italian].

34. Naud P, Matos J, Hammes L et al. Efficacy and tolerability of fenticonazole for bacterial vaginosisi treatment: double-blind study, randomized, prospective, compared to placebo [in Portugese]. Rev. Bras. Med. 60(1-2), 67-70 (2003).

35. Scheres N, Krom BP. Staphylococcus-Candida interaction models: antibiotic resistance testing and host interactions. Methods Mol. Biol. 1356, 153-161 (2016).

36. Bukovsky I, Schneider D, Arieli S, Caspi E. Fenticonazole in the treatment of vaginal trichomoniasis and vaginal mixed infections. Adv. Ther. 8, 166-171 (1991).

37. Fernández-Alba J, Valle-Gay A, Dibildox M et al.; Fentimex Mexican Study Group. Fenticonazole nitrate for treatment of vulvovaginitis: efficacy, safety, and tolerability of 1-gram ovules, administered as ultra-short 2-day regimen. J. Chemother. 16, 179-186 (2004).

38. Antonopoulou S, Aoun M, Alexopoulos EC et al. Fenticonazole activity measured by the methods of the European Committee on Antimicrobial Susceptibility Testing and CLSI against 260 Candida vulvovaginitis isolates from two European regions and annotations on the prevalent genotypes. Antimicrob. Agents Chemother. 53, 2181-2184 (2009).

39. Gorlero F, Sartani A, Cordaro CI, Bertin D, Reschiotto C, De Cecco L. Fenticonazole tissue levels after the application of 3 different dosage forms of vaginal ovules. Intern. J. Clin. Pharm. Ther. Toxicol. 26, 479-481 (1988).

40. Sud IJ, Feingold DS. Action of antifungal imidazoles on Staphylococcus aureus. Antimicrob. Agents Chemother. 22(3), 470-474 (1982).

41. Xie HY, Feng D, Wei DM et al. Probiotics for vulvovaginal candidiasis in non-pregnant women. Cochrane Database Syst. Rev. 11, CD010496 (2017).

42. Bohbot JM, Goubard A, Aubin F et al. PRISM Study: comparison of a nystatin-neomycin-polymyxin B combination with miconazole for the empirical treatment of infectious vaginitis. Med. Mal. Infect. 49(3), 194-201 (2019). 
